\title{
Prediction of the Repair Surface over Cartilage Defects: A Comparison of Three Methods in a Sheep Model
}

\author{
Manuela Kunz ${ }^{1}$, Steven Devlin ${ }^{1}$, Ren Hui Gong ${ }^{1}$, Jiro Inoue ${ }^{1}$, \\ Stephen D. Waldman ${ }^{1}$, Mark Hurtig ${ }^{2}$, Purang Abolmaesumi ${ }^{1}$, \\ and James Stewart ${ }^{1}$
}

1 School of Computing, Department of Materials and Mechanical Engineering,

Department of Surgery, Queen's University, Kingston, ON, Canada

2 Ontario Veterinary College, University of Guelph, Guelph, ON, Canada

\begin{abstract}
Defects in articular cartilage can be repaired through osteochondral transplantation (mosaic arthroplasty), where osteochondral plugs from non-weight-bearing areas of the joint are transferred to the defect site. Incongruity between the plug surface and the adjacent cartilage results in increased contact pressures and poorer outcomes. We compare three methods to predict the desired repair surface for use in computer-assisted mosaic arthroplasty: manual estimation, a cubic spline surface, and a statistical shape atlas of the knee. The cubic spline was found to most accurately match the pre-impact cartilage surface; the atlas was found to match least accurately.
\end{abstract}

\section{Introduction}

Articular cartilage injuries are one of the most common injuries seen in orthopaedic practice. In a review of 31,516 knee arthroscopies, it was found that cartilage defects appeared in $63 \%$ of these cases [1. Damaged articular cartilage in weight-bearing areas of the knee has a large effect on the activity level and life style of the patient [2].

The limited self-healing potential of articular cartilage makes an operative management the common treatment for isolated cartilage defects. One well accepted surgical technique today is osteochondral autograft transplantation. During this procedure, cylindrical osteochondral plugs are retrieved from relatively non-weight-bearing areas of the knee and transplanted into the damaged regions.

For long-term success of this procedure, the transplanted plugs should reconstruct the curvature of the articular surface. Plugs that are placed too high will take a disproportionately large part of the joint load, which may lead to peak loading and abrasion of the plug surface and damage to the opposing articular surface. On the other hand, insufficient plug height results in inappropriate physiological pressure and may be disposed to late degradation [3].

Novel tools [4 and computer assisted procedures [5] have been used to achieve better plug harvesting and placement. But, in the presence of a cartilage defect 
(and the absence of the original cartilage surface), it can be challenging to achieve surface congruity due to lack of a good surface prediction mechanism, particularly in larger defects. Many effective methods have been proposed to segment cartilage from medical images (e.g. 6 6 7 ), but we are not aware of any work to predict the original cartilage surface over a defect site.

The goal of this study was to compare three different artho-CT based methods for determining the desired articular repair surface for the use in computerassisted cartilage repair surgeries. The three methods used manual estimation, a cubic spline, and a statistical shape atlas reconstruction. All methods were tested with an in-vivo sheep model study.

\section{Material and Methods}

Sixteen sheep knees - one from each sheep - were used in the study. For each knee, a CT scan was performed immediately after an intra-articular injection of a contrast agent. All arthro-CTs were obtained with a Light-Speed Plus CT (GE Healthcare, Waukesha, USA) in axial mode, with a slice thickness of 0.625 $\mathrm{mm}$ at $140 \mathrm{kpV}$. The sheep were positioned feet-first supine for the scan.

Three-dimensional surface models for bone and cartilage were manually built using the commercially available software package Amira (Visage Imaging, Inc., Carlsbad, CA, USA). An initial threshold segmentation was performed on the CT volume. This segmentation was manually refined using various editing functions. Isosurface models for bone and cartilage were created and stored.

In a minimally-invasive surgical intervention, a cartilage defect on the medial condyle of each knee was induced with a calibrated impact. Three months after this cartilage damage, a second arthro-CT scan was obtained of each knee in-vivo using the same scanner and protocol as the pre-defect scans. Using the procedure described above, isosurface models for bone and cartilage were created. The three month defects varied in size between $40 \mathrm{~mm}^{2}$ and $60 \mathrm{~mm}^{2}$.

In each of the 16 post-defect CT datasets, the outline of the cartilage defect was manually defined and the desired articular surface over the defect area was predicted using each of three methods: manual estimation; cubic spline surface; and statistical shape atlas. We did not consider using the contralateral knee as a model because the morphology of the two knees is not alway identical, particularly when pathology is present.

The pre-defect and post-defect isosurfaces were co-registered using the iterated closest point (ICP) algorithm [9]. The distance between each predicted surface and the "gold standard" pre-defect isosurface was computed by selecting 50 points on the predicted surface and computing their root-mean-squared error to the pre-defect surface.

\subsection{Method 1: Manual Estimation}

Custom software was developed based on the freely available visualization library Coin3D (www.coin3d.org). For each knee, the post-defect CT volume and 


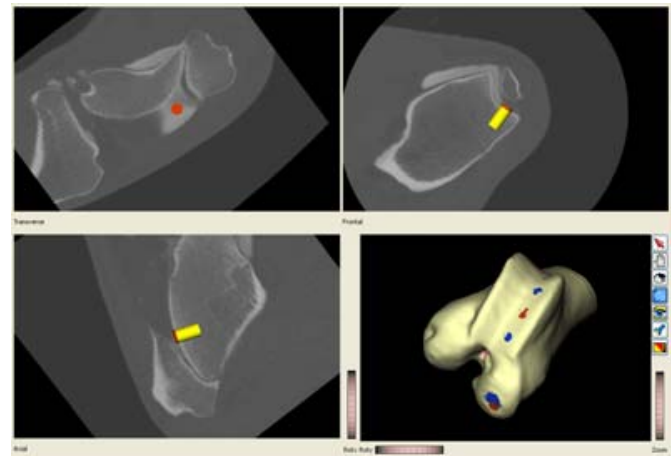

(a)

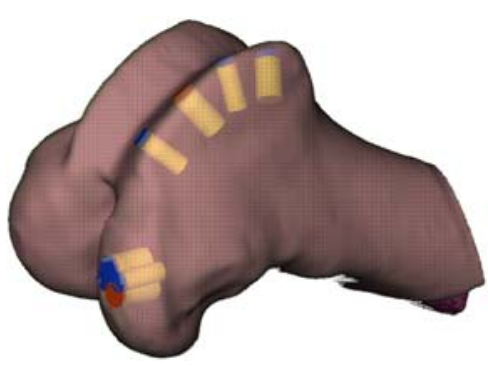

(b)

Fig. 1. (a) Manual planner for cartilage plug placement. The cartilage surface is visible below the light contrast agent in the CT slices. (b) The articular surface over the defect is predicted by the surfaces of the lower plugs on the condyle. (The upper four plugs are the corresponding donor sites.).

the 3D surface models for bone and cartilage were displayed to the operator. Our operator had several years of experience planning orthopaedic surgeries, including mosaic arthroplasty procedures. The operator identified the cartilage defect and created virtual cartilage plugs to reconstruct the articular surface.

For each plug the radius, height, curvature, position and orientation could be modified by the operator. The operator manipulated these parameters to best fit the top of each cartilage plug to what the operator expected would be the pre-defect surface. Each plug could also be shown on the volumetric dataset in three orthographic planes, as shown in Figure 11(a). The operator did not see the pre-defect CT scans during this procedure. The surgeon verified that the planned plugs defined an acceptable articular surface over the defect.

Fifty evenly distributed points were automatically selected on the surfaces of the planned plugs. The resulting points defined the manually estimated cartilage surface and were compared with the gold standard surface.

\subsection{Method 2: Cubic Spline Surface}

A bicubic Hermite surface patch was applied over the defect site. This parametric patch, $Q(s, t)$, was defined by: four points on the surface; two non-orthogonal vectors, $s$ and $t$, describing the direction between these points; the partial derivative with respect to $s$ at each of those four points; the partial derivative with respect to $t$ at each point; and the partial derivative with respect to both $s$ and $t$ at each point (this is called the "twist").

Another operator, who had no previously experience in planning of articular cartilage reconstruction, manually selected the four points $p_{0}, \ldots, p_{3}$ counterclockwise on the post-defect cartilage surface of each knee in a rough rectangle that completely surrounded the defect site, as shown in Figure 2. The vectors 


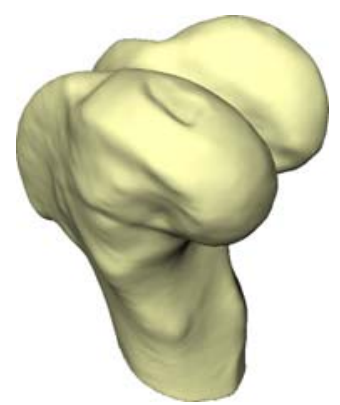

(a)

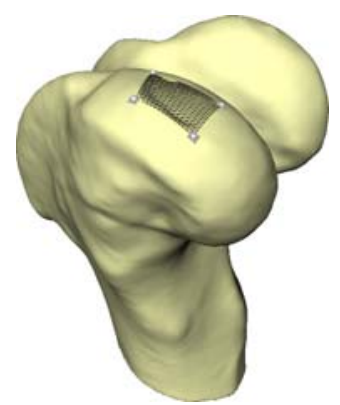

(b)

Fig. 2. (a) Cartilage defect before patch is placed. (b) Four corner points are selected by the operator to define a bicubic Hermite surface patch over the defect site.

$s$ and $t$ were defined as the direction between $p_{0}$ and $p_{1}$ and between $p_{0}$ and $p_{3}$. The partial derivatives at each point were computed using a central finite difference on the scanned cartilage surface, $q$ :

$$
\frac{\delta}{\delta s} q(s, t) \approx \frac{q(s+\Delta s, t)-q(s-\Delta s, t)}{2 \Delta s}
$$

with a $\Delta s$ approximately $5 \%$ of the length of the corresponding edge of the rectangle. The twist vectors were set to zero, which results in a specific type of bicubic Hermite patch, called a Ferguson patch [10]. The surface patch was then constructed in the standard way (e.g. [11]):

$$
Q(s, t)=\left[\begin{array}{c}
F_{1}(s) \\
F_{2}(s) \\
F_{3}(s) \\
F_{4}(s)
\end{array}\right]^{T}\left[\begin{array}{rrrr}
q(0,0) & q(0,1) & q_{t}(0,0) & q_{t}(0,1) \\
q(1,0) & q(1,1) & q_{t}(1,0) & q_{t}(1,1) \\
q_{s}(0,0) & q_{s}(0,1) & q_{s t}(0,0) & q_{s t}(0,1) \\
q_{s}(1,0) & q_{s}(1,1) & q_{s t}(1,0) & q_{s t}(1,1)
\end{array}\right]\left[\begin{array}{c}
F_{1}(t) \\
F_{2}(t) \\
F_{3}(t) \\
F_{4}(t)
\end{array}\right]
$$

with $s, t \in[0,1]$ and $F_{1}(u)=2 u^{3}-3 u^{2}+1, F_{2}(u)=-2 u^{3}+3 u^{2}, F_{3}(u)=$ $u^{3}-2 u^{2}+u$, and $F_{4}(u)=u^{3}-u^{2}$.

For each knee, the fifty points from the manually estimated surface of the same knee were projected onto the bicubic Hermite surface. These fifty projected points were roughly evenly distributed; they were compared to the gold standard surface.

\subsection{Method 3: Statistical Shape Atlas}

A statistical shape atlas was fit to the post-defect isosurface of the distal femur in order to predict the cartilage surface over the defect. The atlas used segmented geometric silhouettes from CT volumes containing the complete femur of 12 sheep cadaver legs (six left and six right). All 12 specimens came from sheep similar in age to the 16 sheep of the study group, but none of the 12 legs was from a sheep in the study group. 
To avoid segmentation errors, all femurs were scanned in air. Each femur was dissected immediately after harvesting, keeping the cartilage on the knee intact. The cartilage was continuously hydrated using a phosphate buffered saline. The femur was scanned using a Light-Speed Plus CT (GE Healthcare, Waukesha, USA) in axial mode, with a slice thickness of $0.625 \mathrm{~mm}$ at $140 \mathrm{kpV}$. The CT images from left legs were mirrored.

Cartilage and bone were manually segmented from each of these training datasets and a binary CT volume (consisting of "inside" and "outside" voxels) was constructed for each knee. We used manual segmentation because the contrast agent is quite prominent in the image and varies substantially in location and shape, making intensity-based registration quite difficult.

The atlas was built in two steps: data normalization and atlas construction. During the data normalization step, all training datasets were transformed into a common coordinate frame and decomposed into a mean shape plus a transformation that determined the variation from the mean shape. This transformation was modeled as the following sequential concatenation of transformations:

1. Rigid transformation for initial alignment: Each femur was reoriented such that its principal axes were aligned with the coordinate axes.

2. Anisotropic scaling for mean size calculation: The scaling factors for each femur were determined using the axis-aligned bounding boxes.

3. Rigid transformation and B-spline deformation for final alignment: A pairwise non-rigid registration was performed to generate the mean shape and calculate the final alignment.

For the atlas construction step, the statistical information of the transformations was captured. For each training dataset, the inverses of the transformations were computed, parameterized, and concatenated to form one column of a matrix, $X$. Since the inverse of a B-spline deformable transform is not analytically available, we approximated the inverse by registering the mean shape back to the training data. Finally, principal component analysis was performed on $X$ and each training dataset, $x_{i}$, was projected into the eigen space, $a_{i}=\operatorname{diag}\left(\delta_{1} \ldots \delta_{12}\right)\left(\left[v_{1} \ldots v_{12}\right]^{T} x_{i}\right)$, where $v_{1}, v_{2}, \ldots, v_{12}$ are the eigenvectors computed from $X X^{T}, \delta_{1}^{2}, \delta_{2}^{2}, \ldots, \delta_{12}^{2}$ are the corresponding variances along each eigenvector, and the $a_{i}$ are the 12-dimensional atlas coefficients corresponding to $x_{i}$. The convex hull of all coefficients contained all valid shapes the atlas could generate, based on the set of training data.

To generate an instance of the atlas, a set of atlas coefficients was provided and the inverse process was performed to compute a sequence of transformations. These transformations were then applied in reversed order to the mean shape. To reduce computational costs, we used only the first eight eigen modes to generate an atlas instance, which accounted for about $85.5 \%$ of total variations in the training data (computed as $\left.\sum_{i=1}^{8} \delta_{i}^{2} / \sum_{i=1}^{12} \delta_{i}^{2}\right)$ ).

To fit the atlas to a specific target knee, the CT volume of the target knee (which had already been segmented) was converted into a binary volume of 


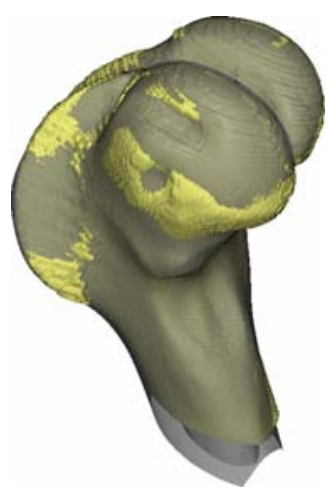

(a)

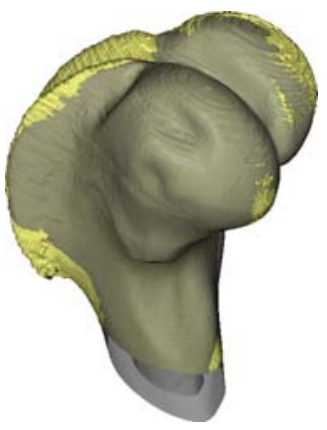

(b)

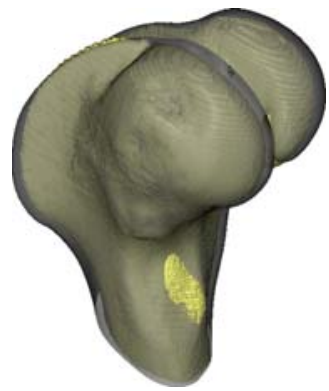

(c)

Fig. 3. Samples of the atlas, as fitted to three different distal femurs. The atlas is shown as transparent gray over the yellow bone-and-cartilage surface. The defect is on the closer condyle of each femur. (a) and (b) show good fits, while (c) shows a poor fit in which the atlas surface is too far above the bone-and-cartilage surface (as seen on the silhouette).

"inside" and "outside" voxels. A rough initial alignment between the atlas mean shape and the target knee CT volume was made manually with a graphical user interface to bring the mean initial alignment RMS error to within $20 \mathrm{~mm}$.

Then the CMA-ES optimization algorithm [12] was used to iteratively and simultaneously (a) refine the initial rigid alignment (6 parameters) and (b) determine the deformation of the atlas ( 8 parameters) using Mutual Information as the similarity metric applied to $5 \%$ (randomly selected) of the voxels of the target knee. The optimization algorithm used the extent of the target knee as the region of interest; the five percent of voxels were taken from this region and were drawn randomly with each iteration. Three sample results are shown in Figure 3 ,

For each knee, the fifty points from the manually estimated surface of the same knee were projected onto the atlas surface; the projected points, which were fairly evenly distributed, were compared to the gold standard surface.

\subsection{Evaluation}

For each of the 16 sheep knees, the cartilage surface was predicted using the three methods described above: manually, using a spline, and using an atlas. Each of these 48 predicted cartilage surfaces was compared to the corresponding true cartilage surface from the pre-defect arthro-CT scan of the same knee.

For each knee, the pre-defect and post-defect bone models were matched with a transformation computed using ICP; the same transformation was applied to the 50 points on each of the three predicted surfaces for that knee. The closest distance between each such point and the cartilage surface of the pre-defect scan was calculated and the RMS error of the 50 points computed. 
Table 1. RMS errors, in mm, of the three methods on 16 knees

\begin{tabular}{l|c|c|c} 
Method & $\begin{array}{c}\text { mean RMS error } \\
95 \% \text { confidence interval }\end{array}$ & $\begin{array}{c}\text { minimum } \\
\text { RMS error }\end{array}$ & $\begin{array}{c}\text { maximum } \\
\text { RMS error }\end{array}$ \\
\hline Spline & $0.27 \pm 0.09$ & 0.10 & 0.54 \\
Manual & $0.30 \pm 0.14$ & 0.08 & 1.04 \\
Atlas & $1.16 \pm 0.33$ & 0.28 & 2.22
\end{tabular}

\section{Results}

Table 1 shows the RMS error between the surface obtained from each of the three methods and the pre-defect surface. The following conclusions were made by applying Student's t-test:

- The spline surface was more accurate than the atlas surface $(p<.0001)$.

- The manual surface was more accurate than the atlas surface $(p<.0001)$.

- The manual and spline surfaces had equivalent accuracy, considering an effect size of $0.25 \mathrm{~mm}$ RMS error to be insignificant $(p<.003)$ (computed using the standard "two one-sided t-tests" method of testing for equivalence).

Also, we observed that the spline surface had a smaller variance in RMS error and a much smaller maximum RMS error than did the manual surface.

We did not measure the time taken to build each surface, but our operators reported the following estimates of time required to construct the predicted cartilage surface from the segmented pre-defect isosurface: 5 minutes for the spline surface; 20 minutes for the manual surface; and 110 minutes for the atlas surface. The manual time estimate included only the time to position the plugs on the defect site. The atlas time estimate used 80 iterations of the optimization procedure.

\section{Discussion}

We conclude that both the manual surface and the spline surface provide accurate reconstructions. The atlas surface, using our particular atlas, does not. The spline surface has several advantages over the manual surface: It is more consistently accurate, has smaller extreme variations, and is faster to use. Our results suggest that the cartilage surface can be well approximated by a cubic spline surface, similar to previous studies (e.g. [8]) that have used spline surfaces to enforce smoothness of the segmentation of an existing cartilage surface.

The poor performance of the atlas might be attributed to the construction or fitting of the atlas itself. More than 12 training knees would yield a better atlas. Restriction of the atlas to just the affected condyle might improve the matching. An atlas built from MR images has been shown [6] to provide excellent cartilage segmentation, and could be extended to predict the cartilage surface over defects. But the spline surface would likely remain superior due to its simplicity of implementation and fast surface construction time. 
The study reported in this paper is part of a larger project on automated planning of computer-assisted mosaic arthroplasty. Any such automatic planner will need a computer representation of the predicted cartilage surface. It appears that a spline surface has the greatest advantages in such a system.

Acknowledgments. This research is supported by grant STPGP 336779-06 from the Natural Sciences and Engineering Research Council of Canada. The authors are grateful to Karen Lowerison, Nicole Kudo and Melissa Lui for their help with data collection and Emily Bishop, David Wright, John Li, Tamara Redwood and Jerome Grondin-Lazzazzera for their valuable help with segmenting the data. We wish to thank the reviewers for their helpful comments.

\section{References}

1. Curl, W., Krome, J., Gordon, E., Rushing, J., Smith, B., Poehling, G.: Cartilage injuries: A review of 31,516 knee arthroscopies. Arthroscopy: The Journal of Arthroscopic and Related Surgery 13(4), 456-460 (1997)

2. Jakob, R., Franz, T., Gautier, E., Mainil-Varlet, P.: Autologous osteochondral grafting in the knee: Indication, results, and reflections. Clinical Orthopaedics and Related Research 401, 170-184 (2002)

3. Koh, J., Wirsing, K., Lautenschlager, E., Zhang, L.Q.: The effect of graft height mismatch on contact pressure following osteochondral grafting: A biomechanical study. American Journal of Sports Medicine 32(2), 317-320 (2004)

4. Brzeczko, A., Goldberg, R., Taylor, R.H., Evans, P.: Smart alignment tool for knee mosaicplasty surgery. In: Medical Image Computing and Computer Assisted Intervention, pp. 599-605 (2001)

5. Hoser, C., Bichler, O., Bale, R., Rosenberger, R., Rieger, M., Kovacs, P., Lang, T., Fink, C.: A computer assisted surgical technique for retrograde autologous osteochondral grafting in talar osteochondritis dissecans (ocd): a cadaveric study. Knee Surgery, Sports Traumatology, Arthroscopy 12(1), 65-71 (2004)

6. Fripp, J., Crozier, S., Warfield, S., Ourselin, S.: Automatic segmentation of articular cartilage in magnetic resonance images of the knee. In: Medical Image Computing and Computer Assisted Intervention, pp. 186-194 (2007)

7. Dam, E., Folkesson, J., Pettersen, P., Christiansen, C.: Semi-automatic knee cartilage segmentation. In: SPIE Medical Imaging: Image Processing, pp. 1286-1294 (2006)

8. Cohen, A., McCarthy, D., Kwak, S., Legrand, P., et al.: Knee cartilage topography, thickness, and contact areas from mri: in-vitro calibration and in-vivo measurements. Osteoarthritis and Cartilage 7, 95-109 (1999)

9. Besl, P., McKay, N.: A method for registration of 3-d shapes. IEEE Transactions on Pattern Analysis and Machine Intelligence 14(2), 239-256 (1992)

10. Ferguson, J.: Multivariable curve interpolation. J. ACM 11(2), 221-228 (1964)

11. Salomon, D.: Curves and Surfaces for Computer Graphics. Springer, Heidelberg (2006)

12. Hansen, N.: The cma evolution strategy: A comparing review. In: Towards a new evolutionary computation. Advances in estimation of distribution algorithms, pp. 75-102. Springer, Heidelberg (2006) 